\title{
Analysis of Relative Risk Factors During Primary and Secondary Stages of Carcinogenesis Causing Growth of a Tumor
}

\author{
Shishir Kumar Jha
}

\begin{abstract}
The objective of this investigation is to find out the relative risks during the primary and the secondary stages of carcinogenesis while affecting the time of growth of a tumor. This result highlights the effectiveness of treatment during the early stage of cancer than that of the same in the advanced stage by reducing hazard rate or postponing the date of appearance of tumor.
\end{abstract}

Key Words: Feller-Arley birth and death process, Hazard rate, Relative Risk, Ricatti equation, Two stage carcinogenesis.

\section{Introduction}

Moolgavakar and Kundson (1981), evolved two stage model of carcinogenesis. They have given the genesis of Tumor cells initially from Normal stem cells; which under the process of primary proliferation give rise to Normal stem cells as well as Initiated (or Intermediate) cells by mutation. Again, the Initiated cells under the secondary proliferation process give rise to Initiated cells and Tumor cell by mutation. They have further assumed that a Tumor cell will cause malignant tumor with probability one. Moolgavkar, Dewanji and Venzon (1988) have extended this two stage model into non-homogenous cases. Tan and Singh (1987) have applied the Moolgavkar Knudson two-stage model to assess effects of metabolism of carcinogens on tumor development.

Assuming that the primary proliferation from Normal to Normal and Initiated cells by mutation take place with probability $\alpha_{1}$ and the secondary proliferation from Initiated cell to Initiated and Tumor cells by mutation occur with probability $\alpha_{2}$, while the birth and death rates of Normal and Initiated cells are time dependent, the objective of the present paper is to investigate the effect of reduction of $\alpha_{1}$ and $\alpha_{2}$ as well as other related time dependent parameters $\left(s a y b_{1}(t), d_{1}(t)\right.$ being the birth and death rates of Normal stem cells and $b_{2}(t), d_{2}(t)$ being corresponding rates for Initiated cells) on the hazard rate or the time of occurrence of a tumor. The motivation of this analysis is to find out to what extent the parameters controlling the growth of the tumor at the primary $\left(\alpha_{1}, b_{1}(t), d_{1}(t)\right)$ stage is more effective than those in secondary $\left(\alpha_{2}, b_{2}(t), d_{2}(t)\right)$ stage in reducing hazard rate or postponing the date of appearance of tumor.

\section{Notations and Assumptions}

Suppose, $n(t), i(t)$ and $x(t)$ denote the number of Normal stem cells, Initiated cells and Tumor cells at time $t$ respectively. On the line of Tan and Brown (1987), the assumptions for developing the model are:

(i) The organ is well developed by time $t_{0}$ (the initial time), so $n(0)\left(=n_{0}\right.$ say) is very large $\left(n_{0} \cong 10^{6}\right.$ to $\left.10^{9}\right)$

(ii) The birth-death processes and the mutation processes are independent of each other.

(iii) $\quad \alpha_{i}(t)=\alpha_{i}, b_{i}(t)=b_{i}$ and $d_{i}(t)=d_{i} ; i=$ 1,2 are independent of $t$.

(iv) We denote by

$$
\begin{gathered}
P_{1}(t)=P_{1}\left(i_{1}, i_{2}, i_{3} ; t\right)=P\left[n(t)=i_{1}, i(t)\right. \\
=i_{2}, x(t)=i_{3} \mid n(0) \\
\left.=n_{0}, i(0)=x(0)=0\right] \\
P_{2}(t)=P_{2}\left(j_{1}, j_{2} ; t\right)=P\left[i(t)=j_{1}, x(t)\right. \\
\left.=j_{2} \mid i(0)=1, x(0)=0\right] \\
\psi(t)=\sum_{i_{1}} \sum_{i_{2}} \sum_{i_{3}} x_{1}^{i_{1}} x_{2}^{i_{2}} x_{3}^{i_{3}} P_{i}(t)
\end{gathered}
$$

\section{Models for primary and secondary stages of Carcinogenesis}

Under the above conditions Tan (1991) has shown that $\varphi(t)$ satisfies the time dependent non-linear Ricatti equation given by

$$
\frac{d}{d t} \varphi(t)=b_{2} \varphi^{2}(t)+\left[\alpha_{2} x_{3}-\left(b_{2}+d_{2}+\right.\right.
$$

$\left.\left.\alpha_{2}\right)\right] \varphi(t)+d_{2}$

subject to the initial condition $\varphi(0)=x_{2}$, and $\alpha_{2}>0$ and $b_{2}>d_{2}>0$

To solve (1), let $\mathrm{a}$ and $\mathrm{b}$ with $\mathrm{b}>\mathrm{a}$ are real numbers for all $x_{3}$ such that

$$
\begin{aligned}
& 2 b_{2} b=\left(b_{2}+d_{2}+\alpha_{2}-\alpha_{2} x_{3}\right)+g\left(x_{3}\right) \\
& 2 b_{2} a=\left(b_{2}+d_{2}+\alpha_{2}-\alpha_{2} x_{3}\right)-g\left(x_{3}\right)
\end{aligned}
$$

and $g\left(x_{3}\right)=\left[\left(b_{2}+d_{2}+\alpha_{2}-\alpha_{2} x_{3}\right)^{2}-4 b_{2} d_{2}\right]^{1 / 2}$

Adding (2) and (3); we obtain

$$
\begin{array}{cc} 
& 2 b_{2}(a+b)=2\left(b_{2}+d_{2}+\alpha_{2}-\alpha_{2} x_{3}\right) \\
\Rightarrow \quad & \alpha_{2} x_{3}=\left(b_{2}+d_{2}+\alpha_{2}\right)-b_{2}(a+b)
\end{array}
$$

Multiplying (2) and (3): we obtain 


$$
d_{2}=b_{2} a b(\text { Using (4)] }
$$

Substituting (5) and (6) in (1); we get

$$
\begin{gathered}
\frac{d}{d t} \varphi(t)=b_{2}[\varphi(t)-a][\varphi(t)-b] \\
\frac{d}{d t} \varphi(t)=b_{2}[\varphi(t)-b+b-a][\varphi(t)-b]
\end{gathered}
$$

Dividing both sides by $(\varphi(t)-b)^{2}$, we get $\frac{1}{[\varphi(t)-b]^{2}} \frac{d}{d t} \varphi(t)=b_{2}+b_{2}(b-a)(\varphi(t)-b)^{-1}$

Put $[\varphi(t)-b]^{-1}=m(t)$ in (8); we obtain

$$
\frac{d}{d t} m(t)+b_{2}(b-1) m(t)=-b_{2}
$$

$\Rightarrow$

$$
e^{b_{2}(b-a) t} \frac{d}{d t} m(t)+b_{2}(b-
$$$$
\text { a)m(t) } e^{b_{2}(b-a) t}=-b_{2} e^{b_{2}(b-a) t}
$$

$\Rightarrow \quad \frac{d}{d t}\left\{m(t) e^{b_{2}(b-a) t}\right\}=-b_{2} e^{b_{2}(b-a) t}$

$\Rightarrow \quad m(t) e^{b_{2}(b-a) t}=-b_{2} \int e^{b_{2}(b-a) t} d t+C$; where $\mathrm{C}$ is constant of integration.

Initially at $\mathrm{t}=0$

$$
\begin{aligned}
& m(0)=[\varphi(0)-b]^{-1}=\left(x_{2}-b\right)^{-1} \\
& \Rightarrow \quad C=\left(x_{2}-b\right)^{-1}+(b-a)^{-1} \\
& \therefore \quad m(t) e^{b_{2}(b-a) t}=(b-a)^{-1}\{1- \\
& \left.e^{b_{2}(b-a) t}\right\}+\left(x_{2}-b\right)^{-1} \\
& =\left[(b-a)\left(x_{2}-b\right)\right]^{-1}\left\{\left(x_{2}-b\right)+(b-\right. \\
& \left.\left.x_{2}\right) e^{b_{2}(b-a) t}+(b-a)\right\} \\
& =\left[(b-a)\left(x_{2}-b\right)\right]^{-1}\left\{\left(x_{2}-a\right)+(b\right. \\
& \left.\left.-x_{2}\right) e^{b_{2}(b-a) t}\right\} \\
& \Rightarrow m(t)=\left[(b-a)\left(x_{2}-b\right)\right]^{-1}\left\{x_{2}-a\right)+ \\
& \left.\left(b-x_{2}\right) e^{b_{2}(b-a) t}\right\}\left\{e^{b_{2}(b-a) t}\right\}^{-1} \\
& \Rightarrow[\varphi(t)-b]^{-1}= \\
& {[(b-a))\left(x_{2}-\right.} \\
& \text { b) } \left.e^{b_{2}(b-a) t}\right]^{-1} \\
& \left.\left[x_{2}-a\right)+\left(b-x_{2}\right) e^{b_{2}(b-a) t}\right] \\
& \Rightarrow \varphi(t)=b+(b-a)\left(x_{2}-b\right) e^{b_{2}(b-a) t}\left\{\left(x_{2}-a\right)+\right. \\
& \left.\left(b-x_{2}\right) e^{b_{2}(b-a) t}\right\}^{-1} \\
& =\left\{b\left(x_{2}-a\right)+a\left(b-x_{2}\right) e^{b_{2}(b-a) t}\right\}\left\{\left(x_{2}-\right.\right. \\
& \left.a)+\left(b-x_{2}\right) e^{b_{2}(b-a) t}\right\}^{-1}
\end{aligned}
$$

Put $\frac{1}{2 b_{2}}\left(b_{2}+d_{2}+\alpha_{2}-\alpha_{2} x_{3}\right)=\mathrm{A}$ and

$$
\frac{1}{2 b_{2}}\left[\left(b_{2}+d_{2}+\alpha_{2}-\alpha_{2} x_{3}\right)^{2}-4 b_{2} d_{2}\right]^{\frac{1}{2}}=B \text { in }
$$

(2) and (3); we obtain

$$
\begin{aligned}
& \mathrm{a}=\mathrm{A}-\mathrm{B} \\
& \mathrm{b}=\mathrm{A}+\mathrm{B}
\end{aligned}
$$

Substituting (10) and (11) in (9); we obtain

$$
\begin{aligned}
\varphi(t)=[(A+B) & \left(x_{2}-A+B\right)+(A-B)(A+B \\
& \left.\left.-x_{2}\right) e^{2 b_{2} B t}\right]\left[\left(x_{2}-A+B\right)+(A+B\right. \\
& \left.\left.-x_{2}\right) e^{2 b_{2} B t}\right]^{-1}
\end{aligned}
$$

Under the assumption that Normal stem cells follow a homogenous Feller-Arley birth and death process (a density dependent birth and death process with birth and death parameters $b_{j}(t)=j b(t)$; and $d_{j}(t)=$ $j d(t) ; \mathrm{j}$ being the size of the population at time $t$ ) with parameters $\left(b_{1}, d_{1}, \alpha_{1}\right) ; \psi(t)$ satisfies the differential equation,

$$
\begin{gathered}
\frac{d}{d t} \psi(t)=b_{1} \psi^{2}(t)+\left[\alpha_{1} \varphi(t)-\left(b_{1}+d_{1}+\right.\right. \\
\left.\left.\alpha_{1}\right)\right] \psi(t)+d_{1}
\end{gathered}
$$

Subject to $n(0)=n_{0}=1$ and $\psi(0)=x_{1}$

Also it is shown by Tan (1991) under the assumption that $n(t) \alpha_{1}(t)$ is finite for all $t \geq t_{0}(=0$, say) and that the number of mutations that occur during $(t, t+\Delta t)$ from Normal stem cells follow a Poison distribution with parameter $n\left(t_{j}\right) \alpha_{1}\left(t_{j}\right) \Delta t+0(\Delta t)$ independently, then

$$
\begin{aligned}
& \begin{array}{l}
\psi\left(t_{0}, t\right)=\psi(t)=e^{\int_{0}^{1} n(x) \alpha_{1}(x)[\varphi(t)-1] d x} \\
{\left[\text { Putting } t_{0}=0\right]}
\end{array} \\
& \text { Put } n(x)=n_{o} e^{\alpha_{1} x_{1}} \quad(14) \\
& \psi(t)=e^{n_{o} \int_{0}^{i} e^{\alpha_{1} x_{1} \alpha_{1}[\varphi(t)-1] d x_{1}}=} \\
& e^{n_{0}[\varphi(t)-1]\left[e^{\alpha_{1} t}-1\right]} \\
& e^{n_{0}\left[\frac{(A+B)\left(x_{2}-A+B\right)+(A-B)\left(A+B-x_{2}\right) e^{2 b_{2} B t}}{\left(x_{2}-A+B\right)+\left(A+B-x_{2}\right) e^{2 b_{2} B t}}-1\right]\left(e^{\alpha_{1} t}-1\right)}
\end{aligned}
$$

Putting $x_{2}-A+B=C$

$A+B-x_{2}=D, A+B=E, A-B=F, 2 b_{2} B=G$, and $2 b_{2} B+\alpha_{1}=H$

in equation (15) with $x_{2}=1$ and $x_{0}=0$, we obtain $\psi(t)=\psi(1,0 ; t)$

$=e^{n_{0}\left[\frac{E C e^{\alpha_{1} t}-E C+F D e^{H t}-F D e^{G t}-C e^{\alpha_{1} t}-D e^{H t}+C-D e^{G i}}{C+D e^{G t}}\right]}$

Differentiating (15) with respect to $\mathrm{t}$ and putting $x_{2}=1$ and $x_{3}=0$; we obtain

$$
\begin{gathered}
\psi^{\prime}(1,0 ; t)= \\
e^{n_{0}\left[\frac{E C e^{\alpha_{1} t}-E C+F D e^{H t}-F D e^{G t}-C e^{\alpha_{1} t}-D e^{H t}+C-D e^{G t}}{C+D e^{G t}}\right]} \times \\
\frac{n_{0}\left[\begin{array}{l}
\left(C+D e^{G t}\right)\left(E C \alpha_{1} e^{\alpha_{1} t}+F D H e^{H t}-F D G e^{G t}-C \alpha_{1} e^{\alpha_{1} t}-H D e^{H t}+D G e^{G t}\right. \\
-\left(E C e^{\alpha_{1} t}-E C+F D e^{H T}-F D e^{G t}-C e^{\alpha_{1} t}-D e^{H t}+C+D e^{G t}\right) D G e^{G t}
\end{array}\right]}{\left(C+D e^{G t}\right)^{2}}
\end{gathered}
$$

Suppose $\lambda(t)$ denotes the hazard rate of growth of tumor given $n_{0}$ Normal stem cells at $t_{o}(=0)$ then Tan (1991) has shown that

$$
\lambda(t)=-\frac{\psi^{\prime}(1,0 ; t)}{\psi(1,0 ; t)}
$$




$$
=\frac{-n_{0}\left[\begin{array}{l}
C e^{\alpha_{1} t}(E-1)\left\{C \alpha_{1}+D e^{G t}\left(\alpha_{1}-G\right)\right\} \\
+D e^{H t}(F-1)\left\{C H+D e^{G t}(H-G)\right\}+C D G e^{G t}(E-F)
\end{array}\right]}{\left(C+D e^{G t}\right)^{2}}
$$

For $x_{2}=1, x_{3}=0$;

$\mathrm{E}-1=\mathrm{D}, \mathrm{F}-1=-\mathrm{C}, \mathrm{H}-\mathrm{G}=\alpha_{1}, \mathrm{E}-\mathrm{F}=2 \mathrm{~B}$

Substituting (21) in (20); we obtain

$$
\begin{aligned}
\lambda(t)=-n_{0}\left[C D e^{\alpha_{1} t}\left\{C \alpha_{1}+D e^{G t}\left(\alpha_{1}-G\right)\right\}\right. \\
-C D e^{\left(G+\alpha_{1}\right) t}\left\{C\left(\alpha_{1}+G\right)\right. \\
\left.+D e^{G t} \alpha_{1}\right\} \\
\left.+2 B C D G e^{G t}\right] \div\left(C+D e^{G t}\right)^{2}
\end{aligned}
$$

Let $\mathrm{T}$ be the time to tumor starting with $\mathrm{n}_{\mathrm{o}}$ Normal stem cells at time $t_{0}$ (suppose $t_{0}=0$ ).

The probability distribution of $\mathrm{T}$ is given by

$$
\begin{aligned}
h_{T}(t) & =\lambda(t) e^{-\int_{0}^{t} \lambda(\tau) d \tau}, t \geq 0 \\
& =0, \text { otherwise }
\end{aligned}
$$

\section{Some special cases for verification of the result}

we should have

$$
\begin{aligned}
& \hat{\lambda}(t)=0 \text { for } \alpha_{1}=0 \\
& \hat{\lambda}(t)=0 \text { for } t=0
\end{aligned}
$$

and $\lambda(t)=0$ for $b_{2}=0 \Rightarrow d_{2}=0, \alpha_{2}=0$

Case I: When $\alpha_{1}=0$

$$
\begin{aligned}
\hat{\lambda}(t) & =\frac{-n_{o}\left(-C D^{2} G e^{G t}-C^{2} D G e^{G t}+2 B C D G e^{G t}\right)}{\left(C+D e^{G t}\right)^{2}} \\
& =\frac{n_{o} C D G e^{G t}(D+C-2 B)}{\left(C+D e^{G t}\right)^{2}}
\end{aligned}
$$

Since, $\mathrm{D}+\mathrm{C}-2 \mathrm{~B}=\mathrm{A}+\mathrm{B}-1+1-\mathrm{A}+\mathrm{B}-2 \mathrm{~B}=0$

Therefore $\alpha_{1}=0 \Rightarrow \lambda(t)=0$

Which should be the case as the hazard rate should tend to zero when $\alpha_{1}=0$

Case II: When $\mathrm{t}=0$

$\hat{\lambda}(t)=\hat{\lambda}(0)$

$$
\begin{aligned}
& =\frac{-n_{o}\left(C D\left(C \alpha_{1}+D \alpha_{1}-D G\right)-C D\left(C \alpha_{1}+C G+D \alpha_{1}\right)+\right.}{(C+D)^{2}} \\
& \qquad \hat{\lambda}(0)=\frac{n_{o}[C D G(C+C-2 B)]}{(C+D)^{2}} \\
& \hat{\lambda}(0)=0 \text { verifies the result for } \mathrm{t}=0 \\
& \text { Case III: } \text { When } b_{2}=0 \\
& \Rightarrow d_{2}=0, \alpha_{2}=0 \\
& \Rightarrow A=1, B=1, C=1, D=1, \text {, and } \mathrm{G}=0 \\
& \Rightarrow e^{G t}=1 \\
& \Rightarrow e^{\left(G+\alpha_{1}\right) t}=e^{\alpha_{1} t} \\
& \therefore \hat{\lambda}(t)=\frac{-n_{0}\left[e^{\alpha_{1} t}\left(\alpha_{1}+\alpha_{1}\right)-e^{\alpha_{1} t}\left(\alpha_{1}+\alpha_{2}\right)+0\right]}{(C+D)^{2}} \\
& =0
\end{aligned}
$$

which should be the case as hazard rate should tend to zero when $b_{2}=0$

\section{Numerical Illustration}

Assuming $b_{2}>d_{2}$ and $\alpha_{1}$ and $\alpha_{2}$ being very small $\left(\alpha_{1}, \alpha_{2} \cong 10^{-6}\right.$ to $\left.10^{-8}\right)$ which is true in most of the cases and further for satisfying the assumption that $n_{0} \alpha_{1}$ is finite, $n_{0}$ should be very large $\left(n_{0} \cong\right.$ $10^{6}$ to $10^{9}$ ).

Let us discuss a particular situation with $b_{2}=.05$ and $d_{2}=.01$. Suppose $\alpha_{1}=\alpha_{2}=.000001$ is one situation naming it as standard situation. Now, we have two experimental situations for comparing the Relative Risks under $10 \%$ increase of $\alpha_{1}$ and $\alpha_{2}$ respectively for different $t$.

Denoting the hazard rate of growth of tumor in the standard situation (i.e. $\alpha_{1}=\alpha_{2}=.000001, b_{2}=$ $\left..05, d_{2}=.01\right)$ at time $t$ by $\lambda_{1}(t)$. Further suppose $\lambda_{2}(t)$ and $\lambda_{3}(t)$ denote the hazard rates under $10 \%$ increase of $\alpha_{1}$ and $\alpha_{2}$ over standard situation respectively. The variations in hazard rates over $\mathrm{t}$ in the above three situations are exhibited in table I.

Table I: Hazards rates over $\mathbf{t}$ with $10 \%$ increase in $\alpha_{1}$ and $\alpha_{2}$ respectively

\begin{tabular}{|l|l|c|c|}
\hline \multirow{2}{*}{$\begin{array}{c}\text { tim } \\
\mathrm{e} \\
(\mathrm{t})\end{array}$} & \multicolumn{3}{|c|}{$\mathrm{b}_{2}=.05, \mathrm{~d}_{2}=.01$} \\
\cline { 2 - 4 } & $\left.\begin{array}{c}\hat{\lambda}_{1}(t) .000001 \\
\alpha_{2}=.000001\end{array}\right)$ & $\left(\begin{array}{c}\hat{\lambda}_{2}(t) \\
\alpha_{2}=.0000011\end{array}\right.$ & $\left.\begin{array}{c}\hat{\lambda}_{3}(t) \\
\alpha_{1}=.000001 \\
\alpha_{2}=.0000011\end{array}\right)$ \\
\hline 1 & $n_{0}(1.2967$ & $n_{0}(1.9061$ & $n_{0}(2.4841$ \\
& $\left.\times 10^{-12}\right)$ & $\left.\times 10^{-12}\right)$ & $\left.\times 10^{-12}\right)$ \\
\hline 2 & $n_{0}(3.7715$ & $n_{0}(4.396$ & $n_{0}(4.5933$ \\
& $\left.\times 10^{-12}\right)$ & $\left.\times 10^{-12}\right)$ & $\left.\times 10^{-12}\right)$ \\
\hline 3 & $n_{0}(5.7807$ & $n_{0}(6.4057$ & $n_{0}(6.615$ \\
& $\left.\times 10^{-12}\right)$ & $\left.\times 10^{-12}\right)$ & $\left.\times 10^{-12}\right)$ \\
\hline 4 & $n_{0}(7.962$ & $n_{0}(8.4211$ & $n_{0}(8.73508$ \\
& $\left.\times 10^{-12}\right)$ & $\left.\times 10^{-12}\right)$ & $\left.\times 10^{-12}\right)$ \\
\hline 5 & $n_{0}(11.1389$ & $n_{0}(12.03$ & $n_{0}(12.233$ \\
& $\left.\times 10^{-12}\right)$ & $\left.\times 10^{-12}\right)$ & $\left.\times 10^{-12}\right)$ \\
\hline 10 & $n_{0}(26.84$ & $n_{0}(28.028$ & $n_{0}(28.9028$ \\
& $\left.\times 10^{-12}\right)$ & $\left.\times 10^{-12}\right)$ & $\left.\times 10^{-12}\right)$ \\
\hline
\end{tabular}

Suppose $\hat{R R_{1}}(t)=\frac{\hat{\lambda_{2}(t)}}{\lambda_{1}(t)}$ denotes the estimates of Relative Risk at time $\mathrm{t}$ because of increasing $\alpha_{1}$ by $10 \%$.

and $\hat{R R}(t)=\frac{\hat{\lambda_{3}(t)}}{\lambda_{1}(t)}$ denotes the estimates of Relative Risk at time t because of increasing $\alpha_{2}$ by $10 \%$. The behaviour of relative risks over time in above two cases is exhibited in table II.

Table II: Relative Risks under $10 \%$ increase of $\alpha_{1}$ and $\alpha_{2}$ respectively for different $t$

\begin{tabular}{|c|c|c|}
\hline \multirow{2}{*}{$\mathrm{t}$} & \multicolumn{2}{|c|}{$b_{2}=.05 d_{2}=.01$} \\
\cline { 2 - 3 } & $\hat{R R_{1}(t)}$ & $\hat{R R_{2}(t)}$ \\
\hline 1 & 1.469962 & 1.915709 \\
\hline
\end{tabular}




\begin{tabular}{|c|c|c|}
\hline 2 & 1.165584 & 1.217897 \\
\hline 3 & 1.108118 & 1.144325 \\
\hline 4 & 1.080154 & 1.120428 \\
\hline 5 & 1.056280 & 1.098223 \\
\hline 10 & 1.044262 & 1.076850 \\
\hline
\end{tabular}

\section{Conclusion}

The findings of the table I and table II clearly show that controlling the primary stage proliferation from Normal to Normal and Initiated cells by mutation is more effective in controlling the growth of tumor than controlling the secondary stage proliferation from Initiated to Initiated and Tumor cells by mutation. It may be seen that with increase of $t$ both $R R_{1}(t)$ and $R R_{2}(t)$ tend to unity which is quite plausible; because malignant tumor is $100 \%$ fatal by assumption, although the relative difference between $\hat{R R}_{2}(t)$ and $\hat{R R_{1}}(t)$ is maintained (i.e. $\hat{R R}_{2}(t)>$ $R R_{1}(t)$ for all finite $t$ ).

\section{References}

1. Biswas, S (1988): Stochastic Processes in Demography and Applications, Wiley.

2. Moolgavkar, S.H. and Knudson, A.G. (1981): Mutation and cancer: A model for human carcinogenesis. Jour. Nat. Cancer Inst. 66, 1037-1052.

3. Moolgavkar, S.H., Dewanji, A and Venzon, D.J. (1988): A Stochastic two stage model for cancer risk assessment I: The hazard function and the probability of tumor. Risk Analysis 3, 383-392.

4. Tan, W.Y. (1991): Stochastic models of carcinogenesis, Marcel Dekker Inc. New York, Basel, Hongkong.

5. Tan, W.Y and Brown, C.C. (1987): A non-homogenous two stage model of carcinogenesis. Mathematical Modelling 9, 631-642.

6. Tan, W.Y. and Singh, K. (1987): On assessing the effects of metabolism of environmental agents on cancer tumor developments by a two stage model of carcinogenesis. Env. Health Perspective 74, 203-2010.

Author: Dr Shishir Kumar Jha is an Associate Professor in the department of Statistics, Ramjas College' University of Delhi since 1991.He has been visiting faculty member of Postgraduate department of Statistics, University of Delhi. Dr Jha has Honours degree in Mathematics. He is M.Sc. in Mathematical Statistics, M.Phil. in Statistics and Ph.D. in Statistics from University of Delhi. He is the recipient of National Merit Scholarship and Junior Research Fellowship (UGC). He is a life member of Indian society for Probability and Statistics. He has been Head (Teacher in charge) of the department of Mathematics and Statistics, Ramjas College, University of Delhi during 2000-02 and 2017-18 and Coordinator/Director of School of Foreign and Indian Languages (2010-12), Ramjas College, University of Delhi besides many other administrative positions in the College/University. He has been members of various academic bodies (e.g. Committee of courses, Committee for curriculum development etc.). of University of Delhi. He has published many research papers in the International and National Journal of repute. His specialisations and areas of interest are Biostatistics, Demography and Population Studies. 